\title{
Gamified Smart Objects for Museums Based on Automatically Generated Quizzes Exploting Linked Data
}

\author{
Alejandro López-Martínez
}

\author{
Carlos A. Iglesias
}

\author{
Álvaro Carrera
}

\begin{abstract}
Museums have become through time the cultural conscience of nations by preserving cultural heritage. Although cultural heritage provides a valuable form of learning about mankind, the display of exhibits might not pique visitors' interest. In order to provide engaging and appealing experiences, museums have integrated a number of technologies in their environments. Particularly, there are promising results with the emerging Internet of Things (IoT) technology, enabling the implementation of smart objects to grant exhibits advanced capabilities. Gamification techniques are also commonly used in cultural heritage scenarios to further draw visitors' attention. On this sense, several museums offer for example interactive question-based games. However, these questions must be periodically renewed and such task is time-consuming given the lack of automation of this procedure.

To address these challenges, this paper proposes a lowmaintenance gamified smart object platform to automate the creation of questions about exhibits by exploiting semantic web technologies. The main contributions of this paper are: (i) the design of a gamified smart object platform for museums based on a trivia quiz app; (ii) the automation of question generation schemes for the proposed game; and (iii) the development of a prototype of the proposed platform in a real-life scenario.

Index Terms-cultural heritage, museums, smart objects, linked data, gamification
\end{abstract}

\section{INTRODUCTION}

Heritage can be described as a mode of cultural production in the present that has recourse to the past [1].This past, mankind's history, is fundamental to our identity as individuals and as members of a society [2]. Such importance arises from the belief that cultural heritage allows people to identify, protect and manage aspects of the past in favour of the present and posterity [3], as well as contributing towards people's sense of cultural identity. In order to show different forms of heritage to current and future generations, museums have become the cultural conscience of nations [4] by providing visitors with learning experiences [5] about their exhibits.

Extensive research has been carried out on the relevance of museums as educational institutions [6] and how should they approach the displays of cultural heritage [7], motivating visitors to effectively engage in museum experiences [8]. Consequently, these institutions are increasingly advocating immersive, first-hand experiences where people are given the chance to look, touch or even experiment with exhibited objects [9], [10]. This method of increasing visitor engagement encourages the use of different technologies, being Internet of Things (IoT) [11] a rather succulent emerging technology to exploit for this purpose. IoT can be defined in terms of smart objects. These are depicted as "autonomous, physical digital objects augmented with sensing/actuating, processing, storing and networking capabilities" [12].

Enriching museum visits with smart objects contributes to an enhanced promotion of cultural heritage because users benefit from more enjoyable cultural experiences. It thus appears preferable to employ multimedia facilities which enable visitors to interact with their surrounding cultural environment and better acquire knowledge [13].

One approach to integrating smart objects into museums is by means of mobile phones acting as nexuses between exhibits and visitors. Combined together they can provide added value to museums through advanced visualization of exhibits or gamification [14] techniques. Question-based gamification methods are representative under this category and one must acknowledge the arduous task of manually creating broad, varied and appealing question sets. Likewise laborious and time-consuming might prove to be the maintenance of smart objects.

A number of works shed light upon large scale question generation methodologies [15]-[17], often resorting to Natural Language Processing (NLP) [18] techniques in order to automate this process. Other lines of research [19]-[23] have explored the feasibility of automatically producing questions for games by harnessing semantic web technologies [24]. These frequently rely on the extraction of information from linked data [25] knowledge graphs, such as Wikipedia's ${ }^{1}$ semantic version, DBpedia ${ }^{2}$. However, the potential of automatic question generation schemes using linked data applied to gamified museum environments has not yet been addressed 
by research. The challenge is therefore to effectively exploit Linked Datasets to automatically yield questions adapted to cultural heritage displayed in museums.

This paper proposes a solution that consists in a gamification platform to enrich and enhance museum experiences. The aim is to provide visitors with more knowledge about the collection of exhibits through an enjoyable learning mechanism. In order to achieve this goal, the platform is based on multiple choice questions that are automatically produced by extracting linked data from DBpedia and mining text descriptions about the exhibits through entity linking tools [26]. An advantage of this automation process is that smart object maintenance will require a considerably reduced time investment for museum owners. The proposed platform has been implemented in a real world scenario. Concretely, in the Telecommunications Museum Professor Joaquín Serna located at Escuela Técnica Superior de Ingenieros de Telecomunicación, Universidad Politécnica de Madrid, Spain.

The rest of this paper is organised as follows. Firstly, an overview of related work in Linked Data, Smart Objects and Gamification in museums is given in Section II. Section III describes the methodology followed in order to automatically produce question sets. Afterwards, Section IV presents the architecture of the proposed gamification platform. Then, Section V elaborates on the implementation of a prototype of the platform at the aforementioned Telecommunications Museum. Finally, Section VI addresses the conclusions drawn from this paper and future lines of work.

\section{BACKGROUND}

This section details the background and related work for the platform introduced in this paper. Firstly, an outline on Linked Data and its applications in automatic question generation schemes is given in Section II-A. Secondly, Section II-B explores the use of Linked Data towards the promotion of cultural heritage in museums. Finally, gamification and smart object-driven strategies employed in museums to promote visits are discussed in Section II-C.

\section{A. Linked Data}

Linked Data was conceived by Berners-Lee [25] and refers to "data published on the Web in such a way that it is machinereadable, its meaning is explicitly defined, it is linked to other external data sets, and can in turn be linked to from external data sets". Data on the Semantic Web [24] are represented following the conventions of Resource Description Framework (RDF) [27] standard, using triples format. Furthermore, RDF data can be queried using the semantics and syntax of standardised and well known RDF query languages, such as SPARQL [28].

Additionally, a set of vocabularies known as ontologies enable to describe, represent and relate terms pursuant to their domain. Typically, each ontology represents an area of concern by providing controlled vocabularies of concepts with machine-readable semantics. There is a large number of widely used ontologies to describe different fields of knowledge.
For example, International Committee for Documentation (CIDOC) Conceptual Reference Model (CRM) [29] ontology has been internationally adopted to enable data interchange of cultural heritage and it has become an ISO standard since 2006 [30].

Provided the machine-readable semantics brought about by Linked Data, one of the many approaches towards harnessing these data consists in the automation of knowledge extraction procedures. A large number of fields enjoy the benefits of such automation, as is the case of question generation schemes.

There is considerable research on methods to exploit Linked Datasets to automatically generate questions, often resulting in some type of quiz-based game. Some of these examples include Clover Quiz [23], Linked Data Movie Quiz (LDMQ) [21] or WhoKnows [22]. Clover Quiz is a multiplayer trivia game for Android that automates the generation of multiple choice questions using SPARQL templates to extract knowledge from DBPedia. Similarly, LDMQ offers an online multiple choice quiz consisting of questions about cinematography. The creation of their game aims to assess the viability of automated Q\&A systems in educational contexts. Although WhoKnows is also based on automatically generated multiple choice quizzes, the objective pursued is to provide an appealing form of ranking DBPedia properties according to their relevance in describing a given entity.

\section{B. Linked Data Towards The Promotion Of Cultural Heritage}

Although increasingly more museums are deciding to publish their holdings as Linked Data, there are few bodies of research that focus on exploiting Linked Data towards the promotion of cultural heritage visits and the creation of valueadded museum experiences.

Kovalenko, Mrabet, Schouten and Sedjovic [31] have designed an application to serve personalised museum tours. This is achieved by displaying information about exhibits which is enriched through live queries to external Linked Data sets (e.g. DBPedia) with the aim of extracting complimentary relevant data regarding the artefact. A recommendation system is also included based on the visitor's profile.

Wang et al. [32] propose a similar platform based on RDF models that recommends exhibits and provides an interactive mobile museum guide comparable to the previous item of research mentioned. Additionally, this platform offers a Tour Wizard that generates online museum tours where visitors can semantically search for artworks or related concepts to add them to the tours.

Unlike both aforementioned articles that exploit Linked Data to conceive an application to provide custom museum tours, Kiourt, Koutsoudis and Pavlidis [33] aim to blend cultural heritage and education through a virtual museum framework based on semantic web and game engine technologies wherein users can tailor virtual 3D exhibitions. However, this framework is not mobile phone based, therefore hindering the possibility to use it throughout a real museum visit. The platform proposed in this paper also combines gamification 
with Linked Data, but it has been designed to be used during visits as our game is conceived for smartphones.

\section{Gamification and Smart Objects in Museums}

Digital games have become an integral part of culture [34]. Their appeal and popularity are primarily attributed to the interactive [35] and competitive [36] qualities of games. Therefore, it comes as no surprise that there is a growing interest in aiming to extrapolate these engaging gaming features to serve other purposes than pure leisure. On this sense, a new term known as gamification has been coined. It can be defined as "an informal umbrella term for the use of video game elements in non-gaming systems to improve user experience $(U X)$ and user engagement" [14].

Amongst the techniques employed by museums to provide more appealing and engaging ways of displaying cultural heritage, we endeavour to delve into the integration of smart objects and gamification methods in museum environments.

There are many museums that employ gamification strategies to entertain visitors during visits. Such is the case, for instance, of the RijksMuseum (Amsterdam) [37] or The British Museum [38]. The former is a family-oriented quest app challenging visitors to solve a number of puzzles about some of the exhibits displayed. The latter offers an app that enables visitors to play a scavenger hunt game, motivating them to search for the required artifacts to learn fun facts about them through questions.

The aforementioned approaches draw visitors' attention to only a narrow selection of their exhibits, thus limiting visitors' museum experience. Provided that the questions displayed have to be manually crafted and updated, it is reasonable to fathom the vast amount of time and resources required to scale their game to include all of the museums' holdings. In contrast to this limitation, the proposed app allows visitors to play questions on any item of their choice. This is achieved by applying automatic quiz generation procedures. As a result, any exhibit can be seamlessly granted with smart capabilities, highlighting the versatile creation of smart objects from museums' holdings.

Regarding smart objects, there are many projects proposing different ways in which they can be integrated in the museum environment. The work published by Biondi et al. [39] presents a platform that relies on Bluetooth beacon devices to automatically display on visitors' smartphones information about exhibits. Chianese et al. [40], [41] carried out a project to enhance sculpture-based exhibitions. By means of Bluetooth sensors, nearby sculptures are detected and displayed in an app. Instead of presenting textual information, the sculpture shown on screen begins to talk about itself as well as recommend other sculptures.

Petrelli et al. [42] introduced a platform referred to as the meSch project. Within this project, different prototypes have been implemented in different museums. One application of their technology [43] consists in creating a magnifying glass that reveals different layers of digital content when visitors point it over exhibits that have been augmented. The meSch project was also extended to create smart replicas [44] in the "Atlantic Wall" exhibition of the Museon in Hague, Netherlands. These replicas are 3D printed models of the original objects. Visitors are offered a simple interaction procedure where they can place replicas on display cases and these play multimedia related to the given exhibit.

\section{GAMified SMART OBJects Platform METHODOLOGY}

The proposed platform aims to provide educational and custom museum experiences by means of smart objects combined with gamification techniques. A crucial aspect of this platform is the automatic generation of questions. Therefore, in order to produce these questions, a methodology has been followed, which is portrayed in Fig. 1. Thus, this section will elucidate the concepts behind the creation of such quiz set. With respect to smart objects, their implementation is more suitably described within the interface and user interaction schemes, which are addressed in Section IV.

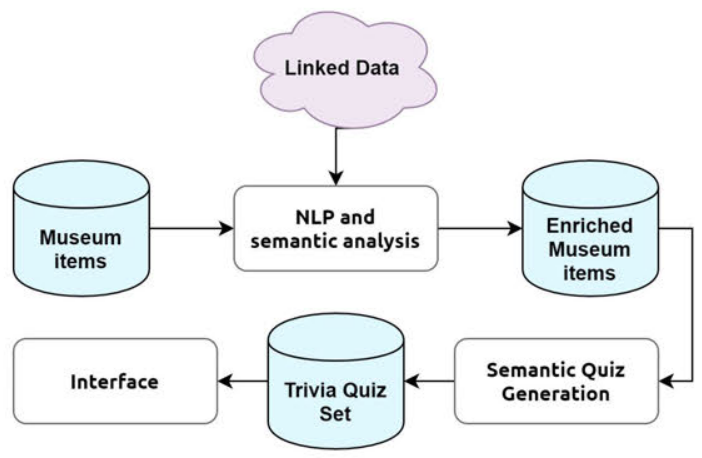

Fig. 1: Methodology for the creation of the Trivia Quiz Set.

Regarding the procedure outlined, there are two principal noteworthy stages: NLP and semantic analysis together with semantic quiz generation. The former process is described in Section III-A; the latter in Section III-B. The ideas conveyed will be illustrated with examples from popular artworks belonging to the renowned Prado Museum ${ }^{3}$ in Spain. Given its popularity and well-documented holdings, we considered it to be an appropriate starting point to assess the viability of the project and methodology in mind.

\section{A. NLP and Semantic Analysis}

Museum exhibits are often complemented with their corresponding captions which briefly describe them. These short descriptions are excerpts from more extensive documentation that can be typically found on museums' online collection. The intention is therefore to extract relevant information from these exhibits through the mining and analysis of these elaborated descriptions. This will be achieved by using NLP techniques and by relying on Linked Datasets to obtain additional relationships about such works.

\footnotetext{
${ }^{3}$ Prado Museum official site: https://www.museodelprado.es/
} 
The Entity Linking process (the semantic annotation process) employs tools that perform Named Entity Recognition (NER) and Named Entity Disambiguation (NED) tasks to locate, classify and distinguish between named entities in the text provided. The disambiguation stage yields matching entries in the Linked Datasets provided. As mentioned in the introduction, DBpedia is the Linked Data knowledge base of choice for our purposes due to its vast amount of entries. To carry out the entity recognition and disambiguation procedures, Babelfy [45] $\mathrm{API}^{4}$ has been selected due to its multilingual capabilities (the text to be processed is written in Spanish) and seamless integration with Wiki- and DB- pedia.

Once results are returned in the form of URLs to the matching DBpedia entries, these have to be classified conforming to the type of entity (category) they represent. Different ontology terms are used to describe diverse properties of entities according to their type. Thus, this categorisation procedure is primordial because only this way can one appropriately query for details of given resources on DBpedia using the right ontology terms. Finally, the classified Linked Entities and documentation about the corresponding museum item are stored.

For instance, complementary information on Velazquez's Las Meninas is to be retrieved from Prado Museum's online collection. An extract from the result of processing this text ${ }^{5}$ with Babelfy API is provided:

\section{"[...]Reflected in the mirror are the faces of Philip IV and Mariana of Austria[... ".}

The underlined concepts are those that have been linked to their corresponding DBpedia entities. The term "mirror" has been identified, hence it can be classified as an object using the schemes described above. A potential question to ask could be for example what other paintings portray mirrors in them. Additionally, the entities linking to King Philip IV of Spain and Queen Mariana of Austria have been recognised. These can be classified as persons, concretely royalty. DBpedia describes people with specific ontology vocabularies, which will be different from the terms used to describe objects (mirrors). Therefore, possible questions that spring to mind could potentially consist in asking about their place and date of birth/death, which can be known by querying specific properties that characterise the set representing people, i.e. $\{$ dbo:birthDate, dbo:deathDate $\in$ dbo:Person.

However, the suggestions of proposed questions are merely illustrative thus far; they have been introduced to inspire the reader to think about possible questions that can be devised using this scheme. Section III-B further elaborates on the quiz generation strategy.

In addition to the aforementioned semantic annotation of text descriptions, another form of obtaining supplementary relevant data related to a given museum item consists in directly resorting to DBpedia. Since the exhibit's documentation

\footnotetext{
${ }^{4}$ Babelfy API official site: http://babelfy.org/

${ }^{5}$ Prado Museums' online documentation about Las Meninas: https://www. museodelprado.es/en/the-collection/art-works?searchObras=las $\ \% 20$ meninas
}

presumably tends to cover aspects such as the authorship and type of work, it is important to shift efforts towards searching in DBpedia for information that is not included in the item's description. Particularly interesting results considering the topics DBpedia encompasses entities in. Referring back to Las Meninas example, the result of the entity search ${ }^{6}$ shows that one of the subjects Velazquez's painting has been classified into is Portraits of Monarchs. Therefore, querying about such topic will yield other works portraying royalty and hence one could prepare questions based on these results. However, the matter of relevance at this stage is the list of subjects presented. These are retrieved from DBpedia and complement the item's documentation. This, together with the already included Linked Entities, form the enriched version of museum items.

Fig. 2 is presented as a means to encapsulate the two main approaches described to extract additional information about museum items.

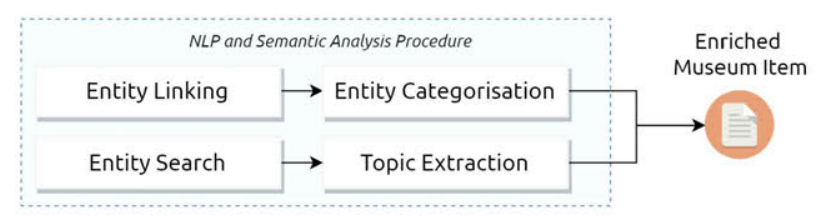

Fig. 2: Tasks involved in the NLP and Semantic Analysis Procedure.

\section{B. Semantic Quiz Generation}

Prior to starting the devising of questions, some considerations shall be taken into account. It must be borne in mind that the quiz game will be displayed in users' smartphones, thereby laying emphasis on producing a mobile friendly interface. A multiple choice-based trivia is therefore an appropriate form of presenting questions in smartphones, allowing users to easily and promptly tap the selected answer instead of having to type it in.

The structure of a Multiple Choice Question (MCQ) consists in the following three items [46]: the stem: the question to formulate; the key: the correct answer; and the distractors: a set of wrong, yet plausible alternatives. Thus, the objective of the semantic quiz generation stage is to produce sets of MCQs according to the described format and in an automated manner.

In order to achieve such automation, MCQs will be generated through question templates. Question templates contain three main elements: the question to formulate; the category, if any, into which the question has been classified; and a parametrised SPARQL query. These, together with the enriched museum data, are fed into the automated question generator. Such generator consists in a procedure that evaluates the annotated data obtained about a museum item against the templates that comply with the specified criteria, i.e. the

\footnotetext{
${ }^{6}$ Las Meninas' structured data version published in DBpedia: http://dbpedia. org/page/Las_Meninas
} 
question category or subject matches the topics of at least one of the pieces of annotated data. Then, the SPARQL query is executed in order to retrieve: (i) the correct answer to the question (the key) and (ii) a list of randomly selected distractors from a set of elements similar to the answer.

With the intention of implementing gamification rules for the Trivia Quiz Game, distractors will be sorted into different sublists according to their difficulty, thus adapting the game to different expertise levels. The suggested approach benefits from the research produced by Zhu and Iglesias [47], which exploits entity similarity scores computed by Sematch ${ }^{7}$ framework in order to classify distractors in consonance with their difficulty. Such scores range from 0 (no apparent similarity between the two named entities) to 1 (strong similarity between them). A total of three sublists of distractors will be generated, corresponding to easy, medium and hard difficulties.

The output of this stage is hence an object containing the enriched museum item together with an array of questions, their answers and the three aforementioned distractor lists. The assignation of the three distractors needed to complete the MCQ is performed in a posterior stage where player's expertise level can be checked. Further details are provided in Section IV.

Referring back to Las Meninas example presented in Section III-A, given the enriched data version produced on this exhibit, it can be, for instance, evaluated against a question template resembling the structure presented in Listing 1.

Listing 1: Example of a simple question template.

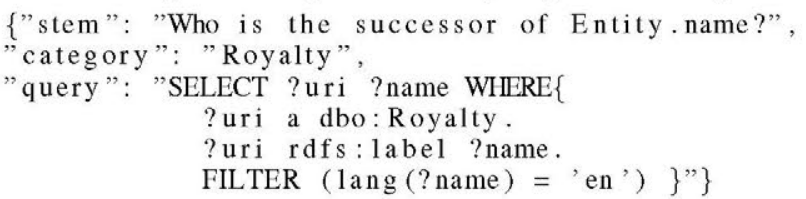

\section{System ARCHITECTURE}

Taking into consideration the question generation methodology outlined in Section III, the resulting architecture consists of the following three main components as depicted in Fig. 3: a data extractor and annotator; a web service and the gaming system.

The data extractor and annotator performs two major tasks. First, this module aims to retrieve museum objects' documentation from their website using web scraping technologies. Second, the extracted data undergoes a semantic annotation procedure and finally these data are fed into a template-based question generator. This yields batches of questions associated to every museum item. The latter procedure has been carried out according to the question generation methodology portrayed in Section III-A.

The web service component stores the information on museum items and their respective generated questions. These are stored as document collections in $\mathrm{MongoDB}^{8}$, the database

\footnotetext{
${ }^{7}$ Sematch is an entity similarity score calculator which has a working demo found in: http://sematch.cluster.gsi.dit.upm.es/

${ }^{8}$ MongoDB official site: https://www.mongodb.com/
}

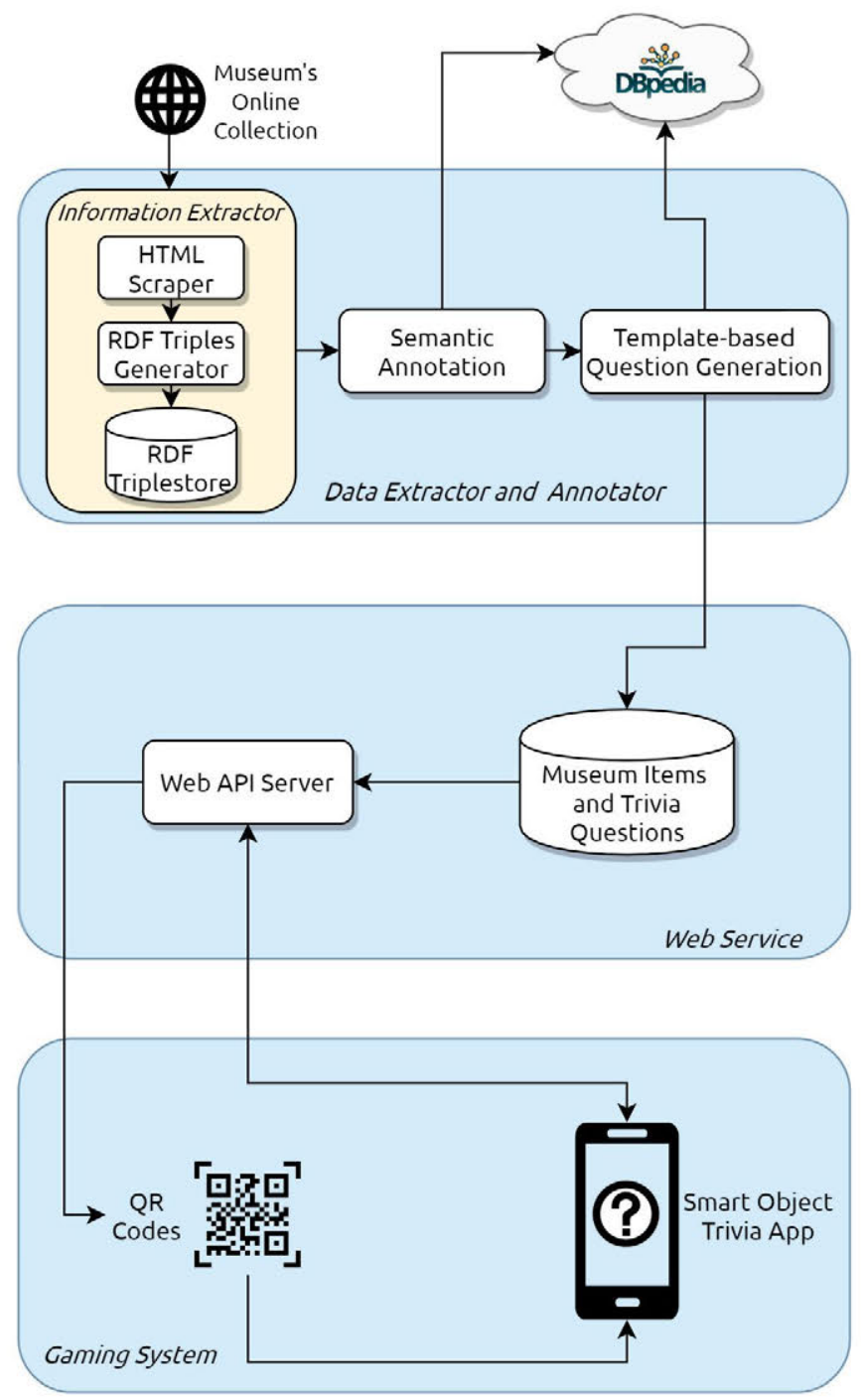

Fig. 3: Gamified Smart Objects Platform Architecture.

of choice because it enables to store documents (NoSQL) in JSON format. Additionally, there is another document to add, which consists of user data and game statistics. The implementation of the web server has been carried out considering REST API paradigms, and it grants the gaming system access to the required resources (object data, questions and user statistics). The server has been developed using Python Flask ${ }^{9}$ web application framework, which is fast and straightforward to set up, whilst providing an excellent performance for our purposes (GET requests only return a JSON-formatted text response). In order to be able to perform the majority of API calls to the service, a valid user token must be used within each request. Such token is granted to a user when they register on our application. For the purpose of improving security, this token is updated each time the user logs in. A demo version

\footnotetext{
${ }^{9}$ Flask official site: https://www.palletsprojects.com/p/flask/
} 
of the web service has been uploaded to Heroku ${ }^{10}$.

Regarding the gaming system, it comprises two main artefacts: QR codes and a smartphone application. These codes contain an $\mathrm{URL}^{11}$ identifying the museum item of interest. Accessing these URLs yield a ISON response containing information about the object. These data are elegantly formatted and presented in the application, so that it provides advanced visualization of the exhibits as well as access to the Trivia quiz game. It must also be noted that images associated to the objects are not stored in our designed database. The main reason is to reduce the load and optimise the speed of the transactions. Instead, we have decided to host them on Cloudinary $^{12}$ servers.

The application has been developed with JavaScript using React Native ${ }^{13}$ framework, granting cross compatibility with Android and iOS.

The gaming system therefore allows the interaction of visitors with the gamified museum environment. The objective of this system is to successfully and effectively accomplish the creation of an interactive museum ecosystem, where QR codes and the designed smartphone application play a critical role.

For the purpose of enhancing the game with motivational affordances, some gamification rules have been devised:

- A score system rewards a number of points between 0 and 10, both inclusive. Players will obtain points by selecting the correct answer within the time limit, and the faster the answer is given, the more points they can be rewarded with, provided the answer picked was the right one. A wrong response does not affect the score.

- Earning points allows registered users to be able to ascend in a ranking system and compare their attained scores with the rest of players by means of a leaderboard.

- As a player makes further progress in the game, questions shown will be harder to adjust for expertise level.

- Tokens can be used throughtout quizzes as a means of additional help. They cross out some of the incorrect answers. These tokens can only be obtained by leveling up and ascending ranks.

\section{A CASE STUDY}

The proposed platform is being implemented in the Telecommunications Museum Professor Joaquín Serna located at Escuela Técnica Superior de Ingenieros de Telecomunicación, Universidad Politécnica de Madrid, Spain. The collection is constituted by 873 telecommunication devices. The platform has been adapted to present information and questions in Spanish.

When endeavouring to perform the entity extraction and annotation procedures outlined in Section III, we came to the realisation that although DBpedia is a large structured

\footnotetext{
${ }^{10}$ Site for demo web service of the proposed system: https://museowebapi. herokuapp.com/

${ }^{11}$ A working example to illustrate this is shown in: https://museowebapi. herokuapp.com/objects/502504

${ }^{12}$ Cloudinary Site: https://cloudinary.com/

${ }^{13}$ React Native Official Website: http://www.reactnative.com/
}

dataset, there are many entities and domains regarding the subject of telecommunications that are missing documentation, not only in Spanish but also in English. This hinders the ability to produce more specific questions about a given object. An example to illustrate this drawback is that the corresponding DBpedia entry about voltmeters ${ }^{14}$ does not describe the different types of voltmeters available or the physical quantity that is being measured. Therefore, a solution to this inconvenience was to classify items into categories in order to generate additional questions related to the theme the object belongs to. As a result, exhibits were classified into four main categories: Sound and Image; Telephony; Radio and Telegraphy; and Instrumentation. Table I portrays the result of such classification. It must be noted that some items belong to more than one theme.

A total of 853 relevant pieces of data could be extracted from items' descriptions and direct DBpedia queries to fill the question templates, as shown in Table II. Given the limitations mentioned, we were able to produce four question templates for each theme. Combined with the extracted data, the result has been the generation of over 200 questions per category.

TABLE I: Breakdown of museum objects into four categories.

\begin{tabular}{|c|c|}
\hline & Number of objects \\
\hline Sound and Image & 72 \\
\hline Telephony & 596 \\
\hline Radio and Telegraphy & $\mathbf{1 4 4}$ \\
\hline Instrumentation & 76 \\
\hline
\end{tabular}

TABLE II: Number of entities per category extracted from exhibits' documentation.

\begin{tabular}{|c|c|}
\hline & Number of extracted entities \\
\hline Sound and Image & 82 \\
\hline Telephony & 490 \\
\hline Radio and Telegraphy & 213 \\
\hline Instrumentation & 68 \\
\hline
\end{tabular}

Moreover, QR codes have been printed into hard cardboards. We prepared a list of $8 \mathrm{QR}$ codes per cardboard, each of them complemented with an image of the exhibit and its name. The choice of format aims to encourage visitors interested in learning more about any museum object to take any of these cardboards and walk around the museum looking for the objects shown in the cardboard. They may scan objects whenever they desire and once they do so, the digital version of the given exhibit will display in app. Not only can visitors play questions about scanned items but they are also provided with additional documentation about them. Hence, the visit shifts towards a treasure-hunt experience. Fig. 4 portrays the final aspect of these hard cardboards.

The smartphone application has been styled and adapted for Joaquin Serna Telecommunications Museum. An example of the app interface is provided in Fig. 5. Concretely, Fig. 5a

\footnotetext{
${ }^{14} \mathrm{DBpedia}$ entry about voltmeters: http://dbpedia.org/page/Voltmeter
} 


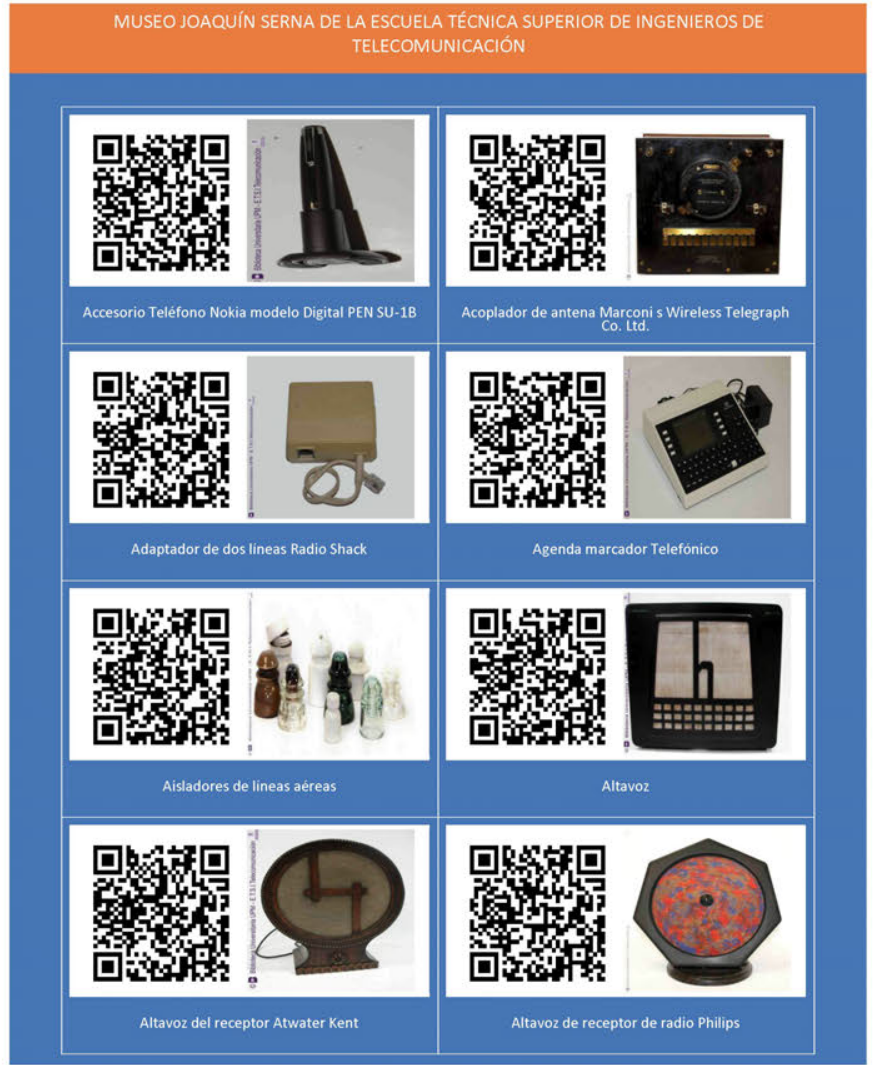

Fig. 4: Cardboard example template used to display QR codes.

shows the representation of an exhibit's documentation in the app. Fig. 5b depicts the user interface of a multiple choice question, where gamification elements can be seen, such as time left and remaining tokens.

\section{CONCLUSIONS AND Future Work}

In this paper, we introduced a smart object gamification platform for museums. The system exploits a combination of semantic web and Internet of Things technologies in order to craft appealing and memorable museum experiences. We have detailed the process by which questions are automatically generated based on a set of templates. Then, we described the interaction of visitors with the museum environment by means of QR codes and a smartphone application that provides advanced visualization and information about the exhibits of interest. The app also offers visitors the opportunity to play the questions that have been generated, thus gamifying their experience. Finally, the prototype of the designed platform was implemented in Joaquín Serna Telecommunications Museum.

Therefore, as future work, an evaluation with museum visitors will be carried out wherein participants answer a number of questions addressing their satisfaction and motivation when using the platform. For reliability, we plan on using a measurement model based on "Partial Least Squares Structural Equation Modeling" [48].

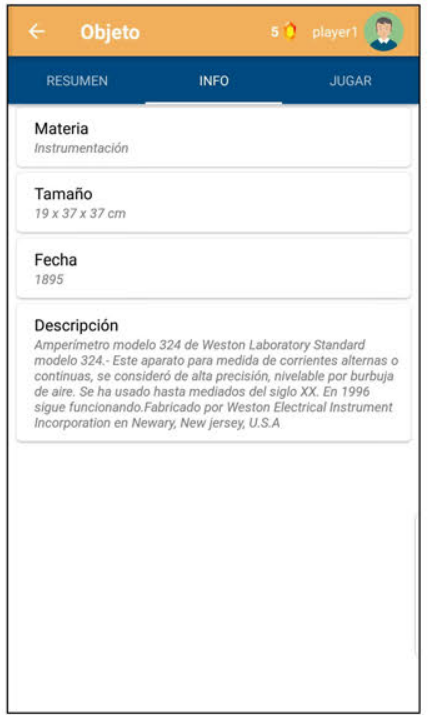

(a) Information of the object displayed in app.

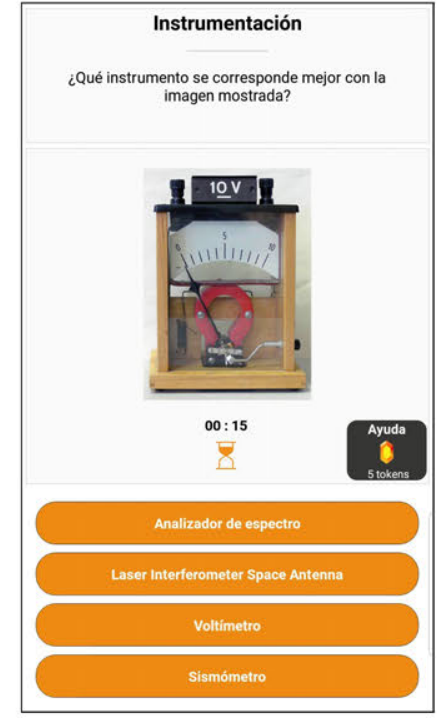

(b) Interface of a multiple choice question.
Fig. 5: Screenshots of the smartphone application

\section{ACKNOWLEDGEMENTS}

The authors express their gratitude to the Library of Escuela Técnica Superior de Ingenieros de Telecomunicación, Universidad Politécnica de Madrid for approving the development of the designed system prototype in Joaquin Serna Telecommunications Museum and for printing hard cardboards containing QR codes.

\section{REFERENCES}

[1] B. Kirshenblatt-Gimblett, Destination culture: Tourism, museums, and heritage. Univ of California Press, 1998.

[2] D. Lowenthal, "Environmental perception: preserving the past," Progress in Geography, vol. 3, no. 4, pp. 549-559, 1979.

[3] M. Pearson and S. Sullivan, Looking after heritage places. Melbourne Univ. Publishing, 2013.

[4] E. N. Arinze, "The role of the museum in society," in Museums, Peace, Democracy and Governance in the 21st Century-Post Conference Workshop, Georgetown, Guyana. Retrieved from http://www. maltwood. uvic. ca/cam/activities/past_conferences/1999conf/CAM, 1999.

[5] G. E. Hein, Learning in the Museum. Routledge, 2002.

[6] E. Hooper-Greenhill, Museums and their visitors. Routledge, 2013.

[7] E. P. Alexander, M. Alexander, and J. Decker, Museums in motion: An introduction to the history and functions of museums. Rowman \& Littlefield, 2017.

[8] J. H. Falk and L. D. Dierking, The museum experience. Routledge, 2016.

[9] —-, Learning from museums. Rowman \& Littlefield, 2018.

[10] K. R. Jeffery-Clay, "Constructivism in museums: How museums create meaningful learning environments," Journal of Museum Education, vol. 23 , no. 1 , pp. 3-7, 1998.

[11] L. Atzori, A. Iera, and G. Morabito, "The internet of things: A survey," Computer networks, vol. 54, no. 15, pp. 2787-2805, 2010.

[12] G. Fortino and P. Trunfio, Internet of things based on smart objects: Technology, middleware and applications. Springer, 2014.

[13] A. Chianese and F. Piccialli, "Designing a smart museum: When cultural heritage joins iot," in 2014 eighth international conference on next generation mobile apps, services and technologies. IEEE, 2014, pp. 300-306.

[14] S. Deterding, M. Sicart, L. Nacke, K. O'Hara, and D. Dixon, "Gamification. using game-design elements in non-gaming contexts," in CHI' 11 extended abstracts on human factors in computing systems. ACM, 2011, pp. 2425-2428. 
[15] E. Sneiders, "Automated question answering using question templates that cover the conceptual model of the database," in International Conference on Application of Natural Language to Information Systems. Springer, 2002, pp. 235-239

[16] I. Aldabe, M. L. De Lacalle, M. Maritxalar, E. Martinez, and L. Uria, "Arikiturri: an automatic question generator based on corpora and nlp techniques," in International Conference on Intelligent Tutoring Systems. Springer, 2006, pp. 584-594.

[17] M. Liu, R. A. Calvo, and V. Rus, "G-asks: An intelligent automatic question generation system for academic writing support," Dialogue \& Discourse, vol. 3, no. 2, pp. 101-124, 2012.

[18] R. Collobert, J. Weston, L. Bottou, M. Karlen, K. Kavukcuoglu, and P. Kuksa, "Natural language processing (almost) from scratch," Journal of machine learning research, vol. 12, no. Aug, pp. 2493-2537, 2011.

[19] C. Bratsas, D.-E. Chrysou, E. Eftychiadou, D. Kontokostas, P. D. Bamidis, and I. Antoniou, "Semantic web game based learning: An i18n approach with greek dbpedia," in LiLe@ WWW, 2012.

[20] M. Foulonneau, "Generating educational assessment items from linked open data: the case of dbpedia," in Extended Semantic Web Conference. Springer, 2011, pp. 16-27.

[21] G. Á. Rey, I. Celino, P. Alexopoulos, D. Damljanovic, M. Damova, N. $\mathrm{Li}$, and V. Devedzic, "Semi-automatic generation of quizzes and learning artifacts from linked data," 2012.

[22] M. Ketterl, L. Knipping, N. Ludwig, R. Mertens, J. Waitelonis, M. Knuth, and H. Sack, "Whoknows? evaluating linked data heuristics with a quiz that cleans up dbpedia," Interactive Technology and Smart Education, 2011.

[23] G. Vega-Gorgojo, "Clover quiz: a mobile trivia game based on dbpedia data." in International Semantic Web Conference (Posters, Demos \& Industry Tracks), 2017.

[24] T. Berners-Lee, J. Hendler, O. Lassila et al., "The semantic web," Scientific american, vol. 284, no, 5, pp. 28-37, 2001

[25] C. Bizer, T. Heath, and T. Berners-Lee, "Linked data: The story so far," in Semantic services, interoperability and web applications: emerging concepts. IGI Global, 2011, pp. 205-227.

[26] X. Han, L. Sun, and J. Zhao, "Collective entity linking in web text: a graph-based method," in Proceedings of the 34th international ACM SIGIR conference on Research and development in Information Retrieval. ACM, 2011, pp. 765-774.

[27] M. Lanthaler, D. Wood, and R. Cyganiak, "RDF 1.1 concepts and abstract syntax," W3C, W3C Recommendation, 2014, http://www.w3.org/TR/2014/REC-rdf11-concepts-20140225/.

[28] A. Seaborne and E. Prud'hommeaux, "SPARQL query language for RDF," W3C, W3C Recommendation, 2008, http://www.w3.org/TR/2008/REC-rdf-sparql-query-20080115/.

[29] M. Doerr, "The cidoc conceptual reference module: an ontological approach to semantic interoperability of metadata," AI magazine, vol. 24 no. 3 , pp. $75-75,2003$.

[30] T. ISO, "46. cidoc coneptual reference model (crm)-iso 21127: 2006," International Standardizaton Organization (ISO), p. 44, 2006.

[31] O. Kovalenko, Y. Mrabet, K. Schouten, and S. Sejdovic, "Linked data in action: Personalized museum tours on mobile devices." in ESWC Developers Workshop, 2015, pp. 14-19.

[32] Y. Wang, N. Stash, R. Sambeek, Y. Schuurmans, L. Aroyo, G. Schreiber, and P. Gorgels, "Cultivating personalized museum tours online and onsite," Interdisciplinary science reviews, vol, 34, no, 2-3, pp. 139-153, 2009.

[33] C. Kiourt, A. Koutsoudis, and G. Pavlidis, "Dynamus: A fully dynamic 3d virtual museum framework," Journal of Cultural Heritage, vol. 22, pp. 984-991, 2016.

[34] "Esa essential facts 2019," https://www.theesa.com/wp-content/ uploads/2019/05/ESA_Essential_facts_2019_final.pdf, (Accessed on $11 / 05 / 2019)$

[35] D. Zillmann and P. Vorderer, Media entertainment: The psychology of its appeal. Routledge, 2000.

[36] P. Vorderer, T. Hartmann, and C. Klimmt, "Explaining the enjoyment of playing video games: the role of competition," in Proceedings of the second international conference on Entertainment computing. Carnegie Mellon University, 2003, pp. 1-9.

[37] "Digital family game - families, education or groups - rijksmuseum," https://www.rijksmuseum.nl/en/family-game, (Accessed on 11/06/2019),

[38] "Gamification of the museum experience - gamification-of-the-museum-experience-ux-case-study-2309d5fd16c3, (Accessed on 11/06/2019).

[39] S. M. Biondi, V. Catania, S. Monteleone, M. Palesi, and D. Patti, "smartworks: A multi-sided context-aware platform for the smart museum," in PECCS, 2018.

[40] F. Amato, A. Chianese, A. Mazzeo, V. Moscato, A. Picariello, and F. Piccialli, "The talking museum project," Procedia Computer Science, vol. 21, pp. 114-121, 2013.

[41] A. Chianese and F. Piccialli, "Improving user experience of cultural environment through iot: the beauty or the truth case study," in Intelligent interactive multimedia systems and services. Springer, 2015, pp. 11-20.

[42] D. Petrelli, M. Marshall, E. Not, M. Zancanaro, A. Venturini, D. Cavada, T. Kubitza, A. Schmidt, M. Risseeuw, and D. Van Dijk, "mesch: Internet of things and cultural heritage," SCIRES-IT-SClentific RESearch and Information Technology, vol, 6, no. 1, pp. 15-22, 2016.

[43] A. Damala, E. Hornecker, M. van der Vaart, D. van Dijk, and I. Ruthven, "The loupe: tangible augmented reality for learning to look at ancient greek art," Mediterranean Archaeology and Archaeometry, vol, 16, no, 5, pp. 73-85, 2016.

[44] M. T. Marshall, N. Dulake, L. Ciolfi, D. Duranti, H. Kockelkorn, and D. Petrelli, "Using tangible smart replicas as controls for an interactive museum exhibition," in Proceedings of the TEI'16: Tenth International Conference on Tangible, Embedded, and Embodied Interaction. ACM, 2016, pp. 159-167.

[45] A. Moro, F. Cecconi, and R. Navigli, "Multilingual word sense disambiguation and entity linking for everybody." in International Semantic Web Conference (Posters \& Demos), 2014, pp. 25-28.

[46] A. Papasalouros, K. Kanaris, and K. Kotis, "Automatic generation of multiple choice questions from domain ontologies." in e-Learning. Citeseer, 2008, pp. 427-434.

[47] G. Zhu and C. A. Iglesias, "Sematch: Semantic similarity framework for knowledge graphs," Knowledge-Based Systems, vol. 130, pp. 30-32, 2017.

[48] M. Tenenhaus, V. E. Vinzi, Y.-M. Chatelin, and C. Lauro, "Pls path modeling," Computational statistics \& data analysis, vol. 48, no. 1, pp. $159-205,2005$. 